\title{
Flow equation renormalization of a spin-boson model with a structured bath
}

\author{
Silvia Kleff ${ }^{a, 1}$, Stefan Kehrein ${ }^{b}$, Jan von Delft ${ }^{a}$ \\ ${ }^{a}$ Lehrstuhl für Theoretische Festkörperphysik, Ludwig-Maximilians Universität, Theresienstr.37, 80333 München, Germany \\ b Theoretische Physik III - Elektronische Korrelationen und Magnetismus, Universität Augsburg, 86135 Augsburg, Germany
}

\begin{abstract}
We discuss the dynamics of a spin coupled to a damped harmonic oscillator. This system can be mapped to a spinboson model with a structured bath, i.e. the spectral function of the bath has a resonance peak. We diagonalize the model by means of infinitesimal unitary transformations (flow equations), thereby decoupling the small quantum system from its environment, and calculate spin-spin correlation functions.
\end{abstract}

Key words: flow equations; quantum dissipative systems, spin-boson, structured bath

\section{Introduction - Model}

Recently a new strategy for performing measurements on solid state (Josephson) qubits was proposed which uses the entanglement of the qubit with states of a damped oscillator [1], with this oscillator representing the plasma resonance of the Josephson junction. This system of a spin coupled to a damped harmonic oscillator (see Fig. 1) can be mapped to a standard model for dissipative quantum systems, namely the spin-boson model [2]. Here the spectral function governing the dynamics of the spin has a resonance peak. Such structured baths were also discussed in connection with electron transfer processes [2]. We use the flow equation method introduced by Wegner [3] to analyze the system shown in Fig. 1, consisting of a two-

1 E-mail: kleff@theorie.physik.uni-muenchen.de

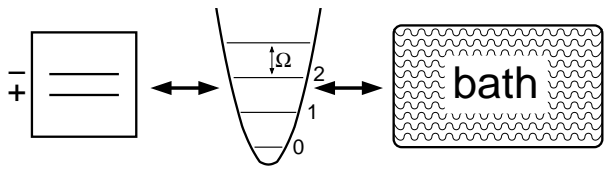

Fig. 1. A two-level system is coupled to a damped harmonic oscillator with frequency $\Omega$. level system coupled to a harmonic oscillator $\Omega$, which is coupled to a bath of harmonic oscillators:

$$
\begin{aligned}
\tilde{\mathcal{H}} & =-\frac{\Delta_{0}}{2} \sigma_{\mathrm{x}}+\Omega B^{\dagger} B+g\left(B^{\dagger}+B\right) \sigma_{\mathrm{z}}+\sum_{\mathrm{k}} \tilde{\omega}_{\mathrm{k}} \tilde{b}_{\mathrm{k}}^{\dagger} \tilde{b}_{\mathrm{k}} \\
& +\left(B^{\dagger}+B\right) \sum_{\mathrm{k}} \kappa_{\mathrm{k}}\left(\tilde{b}_{\mathrm{k}}^{\dagger}+\tilde{b}_{\mathrm{k}}\right)+\left(B^{\dagger}+B\right)^{2} \sum_{\mathrm{k}} \frac{\kappa_{\mathrm{k}}^{2}}{\tilde{\omega}_{\mathrm{k}}}
\end{aligned}
$$

with the spectral function $J(\omega) \equiv \sum_{\mathrm{k}} \kappa_{\mathrm{k}}^{2} \delta\left(\omega-\tilde{\omega}_{\mathrm{k}}\right)=$ $\Gamma \omega$. This system can be mapped to a spin-boson model [2]

$$
\mathcal{H}=-\frac{\Delta_{0}}{2} \sigma_{\mathrm{x}}+\frac{1}{2} \sigma_{\mathrm{z}} \sum_{\mathrm{k}} \lambda_{\mathrm{k}}\left(b_{\mathrm{k}}^{\dagger}+b_{\mathrm{k}}\right)+\sum_{\mathrm{k}} \omega_{\mathrm{k}} b_{\mathrm{k}}^{\dagger} b_{\mathrm{k}},
$$

where the dynamics of the spin depends only on the spectral function $J(\omega) \equiv \sum_{\mathrm{k}} \lambda_{\mathrm{k}}^{2} \delta\left(\omega-\omega_{\mathrm{k}}\right)$ given by

$J(\omega)=\frac{2 \alpha \omega \Omega^{4}}{\left(\Omega^{2}-\omega^{2}\right)^{2}+(2 \pi \Gamma \omega \Omega)^{2}}$ with $\alpha=\frac{8 \Gamma g^{2}}{\Omega^{2}}$.

\section{Method - Results}

Using the flow equation technique we approximately diagonalize the Hamiltonian $\mathcal{H}$ [Eq.(1)] by means of 
infinitesimal unitary transformations. The continuous sequence of unitary transformations $U(l)$ is labelled by a flow parameter $l$. Applying such a transformation to a given Hamiltonian, this Hamiltonian becomes a function of $l: \mathcal{H}(l)=U(l) \mathcal{H} U^{\dagger}(l)$. Here $\mathcal{H}(l=0)=\mathcal{H}$ is the initial Hamiltonian and $\mathcal{H}(l=\infty)$ is the final diagonal Hamiltonian. Usually it is more convenient to work with a differential formulation

$$
\frac{d \mathcal{H}(l)}{d l}=[\eta(l), \mathcal{H}(l)] \quad \text { with } \quad \eta(l)=\frac{d U(l)}{d l} U^{-1}(l) .
$$

Using the flow equation approach one can decouple system and bath by diagonalizing $\mathcal{H}(l=0)$ [4]:

$\mathcal{H}(l=\infty)=-\frac{\Delta_{\infty}}{2} \sigma_{\mathrm{x}}+\sum_{\mathrm{k}} \omega_{\mathrm{k}} b_{\mathrm{k}}^{\dagger} b_{\mathrm{k}}$

Here $\Delta_{\infty}$ is the renormalized tunneling frequency. For the generator of the flow we choose the Ansatz [4]:

$$
\begin{aligned}
\eta & =\sum_{\mathrm{k}}\left(\mathrm{i} \sigma_{\mathrm{y}} \Delta\left(b_{\mathrm{k}}+b_{\mathrm{k}}^{\dagger}\right)+\sigma_{\mathrm{z}} \omega_{\mathrm{k}}\left(b_{\mathrm{k}}-b_{\mathrm{k}}^{\dagger}\right)\right) \frac{\lambda_{\mathrm{k}}}{2}\left(\frac{\Delta-\omega_{\mathrm{k}}}{\Delta+\omega_{\mathrm{k}}}\right) \\
& +\frac{\Delta}{2} \sum_{\mathrm{q}, \mathrm{k}} \lambda_{\mathrm{k}} \lambda_{\mathrm{q}} I\left(\omega_{\mathrm{k}}, \omega_{\mathrm{q}}, l\right)\left(b_{\mathrm{k}}+b_{\mathrm{k}}^{\dagger}\right)\left(b_{\mathrm{q}}-b_{\mathrm{q}}^{\dagger}\right)
\end{aligned}
$$

with $I\left(\omega_{\mathrm{k}}, \omega_{\mathrm{q}}, l\right)=\frac{\omega_{\mathrm{q}}}{\omega_{\mathrm{k}}^{2}-\omega_{\mathrm{q}}^{2}}\left(\frac{\omega_{\mathrm{k}}-\Delta}{\omega_{\mathrm{k}}+\Delta}+\frac{\omega_{\mathrm{q}}-\Delta}{\omega_{\mathrm{q}}+\Delta}\right)$.

The flow equations for the effective Hamiltonian [Eq. (4)] then take the following form:

$$
\begin{aligned}
\frac{\partial J(\omega, l)}{\partial l} & =-2(\omega-\Delta)^{2} J(\omega, l) \\
& +2 \Delta J(\omega, l) \int \mathrm{d} \omega^{\prime} J\left(\omega^{\prime}, l\right) I\left(\omega, \omega^{\prime}, l\right), \\
\frac{d \Delta}{d l} & =-\Delta \int \mathrm{d} \omega J(\omega, l) \frac{\omega-\Delta}{\omega+\Delta} .
\end{aligned}
$$

The unitary flow diagonalizing the Hamiltonian generates a flow for $\sigma_{\mathrm{z}}(l)$ which takes the structure

$\sigma_{\mathrm{z}}(l)=h(l) \sigma_{\mathrm{z}}+\sigma_{\mathrm{x}} \sum_{\mathrm{k}} \chi_{\mathrm{k}}(l)\left(b_{\mathrm{k}}+b_{\mathrm{k}}^{\dagger}\right)$,

where $h(l)$ and $\chi_{\mathrm{k}}(l)$ obey the differential equations

$$
\begin{aligned}
\frac{d h}{d l} & =-\Delta \sum_{\mathrm{k}} \lambda_{\mathrm{k}} \chi_{\mathrm{k}} \frac{\omega_{\mathrm{k}}-\Delta}{\omega_{\mathrm{k}}+\Delta} \\
\frac{d \chi_{\mathrm{k}}}{d l} & =\Delta h \lambda_{\mathrm{k}} \frac{\omega_{\mathrm{k}}-\Delta}{\omega_{\mathrm{k}}+\Delta}+\sum_{\mathrm{q}} \chi_{\mathrm{q}} \lambda_{\mathrm{k}} \lambda_{\mathrm{q}} \Delta I\left(\omega_{\mathrm{k}}, \omega_{\mathrm{q}}, l\right) .
\end{aligned}
$$

One can show that the function $h(l)$ decays to zero as $l \rightarrow \infty$. Therefore the observable $\sigma_{\mathrm{z}}$ decays completely into bath operators [4].

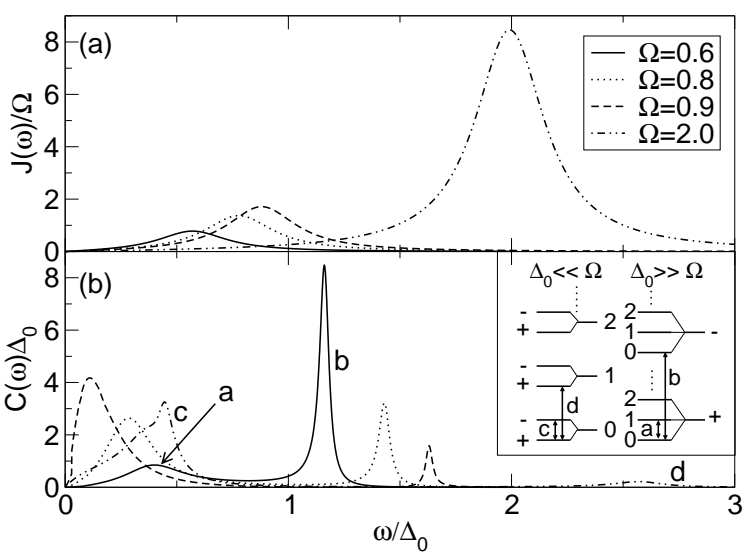

Fig. 2. (a) Different effective spectral functions $J(\omega, l=0)$ and (b) the corresponding $C(\omega)$ for $\Omega \Gamma=0.06$ and $\alpha=0.15$. The inset shows the term scheme of a two-level system coupled to a harmonic oscillator for the two limits $\Delta_{0} \ll \Omega$ and $\Delta_{0} \gg \Omega$.

We integrated the flow equations numerically in order to calculate the Fourier transform, $C(\omega)$, of the spin-spin correlation function

$C(t) \equiv \frac{1}{2}\left\langle\sigma_{\mathrm{z}}(t) \sigma_{\mathrm{z}}(0)+\sigma_{\mathrm{z}}(0) \sigma_{\mathrm{z}}(t)\right\rangle$.

$C(t)$ can be used to calculate dephasing and relaxation times for measurements on qubits [1]. Fig. 2(a) shows $J(\omega, l=0)$ and Fig. 2(b) $C(\omega)$ for different values of $\Omega$. $C(\omega)$ displays a double-peak structure, which can be understood from the term scheme shown in the inset. The arrows indicate the transitions responsible for the peaks in $C(\omega)$. Additional structure of $C(\omega)$ due to higher order transitions in the term scheme is not seen in Fig. 2. This is due to our Ansatz for $\sigma_{\mathrm{z}}(l)$ [see Eq.(8)], which does not include the corresponding higher order terms. However, we do not expect the additional peaks to have much weight, as the sum rule [4] for the total spectral weight is fulfilled with an error of less than $5 \%$ for all the plots in Fig. 2(b). We leave the extension of the Ansatz for $\sigma_{\mathrm{z}}(l)$ for future work.

Acknowledgements The authors would like to thank F. Wilhelm for helpful discussions. S. Kehrein acknowledges support by the SFB 484 of the Deutsche Forschungsgemeinschaft.

\section{References}

[1] F.K. Wilhelm, preprint.

[2] A. Garg et al., J. Chem. Phys. 83, 3391 (1985). 
[3] F. Wegner, Ann. Phys. 3, 77 (1994).

[4] S. Kehrein and A. Mielke, Ann. Phys. 6, 90 (1997). 